\title{
Capitalismo transnacional y desarrollo nacional ${ }^{1}$
}

\author{
INTRODUGCION
}

Durante los últimos tres decenios en la mayor parte de los países denominados subdesarrollados se ha extendido y profundizado notablemente el proceso de desarrollo capitalista. Dicho proceso ha estado condicionado por las características socioculturales y geopolíticas preexistentes en cada país, así como por la naturaleza desequilibrada del propio desarrollo capitalista. Pero además ha sido poderosamente influenciado por las nuevas características que ha adquirido el capitalismo en el período de la postguerra en los paises industrializados.

El enfoque centro-periferia ha sido muy útil para explicar los procesos históricos del desarrollo capitalista en la periferia en función de las características de los centros correspondientes y de sus etapas de expansión y crisis (Prebisch, 1949; CEPAL, 1951; Furtado, 1970; Sunkel y Paz, 1970; Emanuel, 1972; Amin, 1973; Pinto y Kñakal, 1973; Pinto, 1976)². Así, por ejemplo, se reconoce que la difusión de la Revolución Industrial y la

\section{Nota}

El presente trabajo pretende reflejar, en forma muy resumida el estado de nuestras reflexiones acerca del proceso de transnacionalización. Se basa en las investigaciones que se iniciaron hace años y que prosiguen actualmentc. Su objetivo principal es esbozar un marco conceptual coherente al que puedan vincularse trabajos adicionales sobre los numerosos y complejos aspectos del desarrollo. Una investigación tan amplia como esta no podría llevarse a cabo si no se contase con el apoyo sostenido de numerosos colegas $c$ instituciones. En especial, deseamos expresar nuestro reconocimiento a los integtantes del Grupo de Estudios de Dependencia del Institute of Development Studies de la Universidad de Sussex y a los participantes del Seminario sobre Desarrollo Nacional y Relaciones Internacionales, de la misma institución. La colaboración que prestaron Stepanka Griffith-Jones, Rogelio Pérez Perdomo, Arturo Valenzuela y José Villarni en la elaboración del presente trabajo no solo fuc inapreciable sino también muy grata. Como es natural, ellos no comparten necesariamente todo su contenido. En lo que tespecta al apoyo institucional y financiero a esta línea de investigaciones, que yz se prolonga por vatios 2 ños $y$ ha involucrado numerosas personas, estamos sumamente reconocidos con el Institute of Development Studies, e! Institut fur lberoamerika Kunde, la Fundación Ford, la Stiftung Volkswagen y la Agencia Succa para la Cooperación Académica con los países en Desarrollo (SAREC).

${ }^{\mathrm{i}} \mathrm{La}$ versión original en inglés de este articulo aparece en: J.J. Villamil (Ed.) Transnational Capitalism and National Development, Harvester Press, Hassocks uk, 1979.

${ }^{2}$ Aunque en este trabajo se incluyen numerosas referencias a obras que representan diversos intentos de abordar algunos de los múltiples aspectos tratados, conviene aclarar que ello no constituye una biliografia completa y sistemática. 
expansión imperialista de los países centrales durante la última parte del siglo XIX, es el elemento que más ha contribuido a convertir los países periféricos en exportadores especializados de productos primarios. Por otra parte, se acepta que el período de crisis por el que atravezó el capitalismo desde la primera guerra mundial hasta los años cuarenta fue un factor determinante del proceso de industrialización de muchos países subdesarrollados en esa época. Sin embargo, salvo en el sentido restringido de la importancia que revisten los mercados externos de productos básicos, tecnología y capital, y del crecimiento de las empresas transnacionales en la postguerra, que se ha destacado en la literatura sobre la dependencia, el análisis del proceso de desarrollo de los países periféricos en las últimas dos décadas se lleva frecuentemente a cabo como si aquel marco capitalista global hubiese, dejado de existir, hubiese permanecido esencialmente invariable o careciese de importancia ${ }^{3}$.

El presente artículo sugiere, por el contrario que el proceso de desarrollo de las sociedades nacionales en los últimos tres decenios se ha visto condicionado en gran medida por la aparición de un nuevo tipo de sistema global originado por la expansión - mundial del capitalismo oligopólico tecnoindustrial en su nueva etapa de organización transnacional (Sunkel, 1970; Keohane y Nye, 1971; Amin, 1972, Meister, 1976; Michalet, 1976; Mennis y Sauvant, 1976; Fröbel, Heinrichs y Kreye, 1977, Villamil, 1979 a). Por lo tanto el marco dentro del cual hay que concebir el desarrollo nacional en los últímos tres decenios es este nuevo sistema global. Sin embargo, los análisis más convencionales del desarrollo nacional reciente no sólo han pasado por alto con frecuencia las nuevas relaciones entre el centro y la periferia, sino también las profundas modificaciones que se han producido en el propio centro, y sus consecuencias.

En parte como resultado de lo anterior, las políticas nacionales de desarrolio han tendido a subestimar la influencia y la fuerza del capitalismo transnacional. La mayoría de los países han logrado tasas promedio de crecimiento económico y modernización sin precedentes. Al mismo tiempo la naturaleza singular que ha adquirido el proceso de innovación tecnológico, que es el nucleo dinámico de la expansión del capitalismo oligopólico tecnoindustrial, ha tenido consecuencias extremadamente perjudiciales, que rebasan con mucho las desigualdades características del desarrollo capitalista tradicional. En los países subdesarrollados, esta nueva modalidad de crecimiento se traduce en la importación masiva de novedosas formas de consumo, producción, insumos materiales, tecnologia, instituciones, recursos humanos y cultura, que incorporan

\footnotetext{
${ }^{3}$ Véanse, por ejemplo, los conocidos textos de Higgins, Meicr y Baldwin, y G. Meier. La literatura sobre la dependencia fue una reacción a esta situación; véanse por cjemplo los artículos de Girvan (1973), Cardoso (1974), O'Brien (1975), Cueva (1976), Valenzuela y Valenzuela (1979), y Sunkel (1977). En lo que respecta al crecimiento de las empresas transnacionales y sus efectos, véanse Sunkel (1967 y 1970), Vernon (1971), Naciones Unidas (1973 y 1974), Vaitsos (1974).
} 
nuevas estructuras económicas, socioculturales y políticas internas a las antiguas modalidades de dependencia externa, y que acentúan la tendencia estructural a aumentar el desequilibrio exterior (Sunkel, 1967; Furtado 1973; Sauvant y Mennis, 1977). Cuando se dan un elevado y creciente coeficiente de capital, una distribución muy desigual del ingreso y una escasez relativa de ahorro y mercados, la acumulación de capital tiende a concentrarse, con gran desperdicio de recursos escasos, en la producción de nuevos bienes y servicios de consumo, dando lugar a un reducido número de empleos adicionales, a la par que las actividades preexistentes se ven privadas de capital y se estancan, decaen y desaparecen. Esto contribuye al desplazamiento masivo de la mano de obra (Nun, 1969, Quijano, 1974; Godfrey, 1977), y aumenta los scctores marginales e informales, el desempleo, el subempleo, y por consiguiente la pobreza y la desigualdad (Sunkel, 1970. Adelman y Morris, 1973; Chenery y otros 1974, Foxley, 1974).

El presente trabajo procura esbozar en forma sumaria y provisional la naturaleza de este nuevo sistema global, sus principales componentes y sus interrelaciones, sus raíces históricas en la crisis de una fase previa de organización global del capitalismo industrial, y finalmente, la forma en que su presencia condiciona el proceso de desarrollo de las sociedades nacionales.

Conviene advertir de entrada que, dada la naturaleza y objetivos del presente trabajo, se tiende a poner, un acento exagerado en las características sistémicas del capitalismo transnacional y se pasa por alto el análisis importantísimo de sus contradicciones y limitaciones. Además, al centrarse en el proceso de integración de los paises subdesarrollados en el sistema transnacional, fenómeno común a todos ellos, no se analizan las diferencias que surgen en cada caso, que pueden ser muy grandes. Estos son dos importantes temas para investigacion es post eriores.

\section{La Naturaleza del CAPITAlismo TRANSNacional}

El estudio del capitalismo transnacional exige definir una serie de conceptos que se estiman útiles para estos fines.

Calificar el capitalismo transnacional como un sistema, equivale a decir que pese a que el capitalismo contemporáneo, nacional, subnacional y transnacional, se compone de distintas partes, algunas en conflicto y contradicción con otras, todas son estrechamente interdependientes y funcionan sobre la base de parámetros, criterios y objetivos dominantes, actuando y reaccionando como un todo integral. Como es natural,

\footnotetext{
'Otros autores, han sugerido analizar el capitalismo mundial en su fase actual partiendo de conjuntos de conceptos alternativos. Asi Murray (1971), Amin (1972), Palloix (1973), Frobel et al. (1977), hablan de la "internacionalización del capital", mientras Keohane y Nye (1971) y otros se refieren a la creciente "inter dependencia" del sistema internacional.
} 
las formas de incorporación al sistema y las características de cada componente varían de pais a país. Además el comportamiento dentro del sistema tiene cierta flexibilidad, pero hay límites más allá de los cuales un país comienza a verse sometido a fuertes presiones internas y externas y tiene que adaptarse al sistema o quebrar con él.

Se dice que este sistema de tecnoindustrial para referirse a la estrecha interrelación estructural que existe entre la investigación cientîfica pura y aplicada y la producción y la comercialización en gran escala. En esta interrelación reside la esencia de su extraordinario dinamismo tecnológico que le permite producir una gama cada vez más amplia y variada de medios de producción, consumo y destrucción (Galbraith, 1967, Touraine, 1976).

Es oligopólico porque la mayor parte de la. actividad económicaproducción, comercialización, innovación, acumulación, exportaciones, importaciones, etc. se concentra marcadamente en un pequeño número de gigantescas empresas que ocupan una posición central y dominante en relación a un gran número de empresas medianas y pequeñas, responsables de una reducida proporción de la actividad económica total (Senado de los Estados Unidos, 1964 y 1977). También, porque la competencia oligopólica en lugar de operar a través de los precios, tiende a adoptar la forma de la innovación tecnológica y la diferenciación de productos, lo que constituye a su vez el motor que mueve la manipulación de la demanda y del consumidor (Scherer, 1971; Schiller, 1976, Sauvant y Mennis, 1977).

Decir que es capitalista indica que la producción se organiza sobre la base de la propiedad privada de los medios de producción, la maximización de las utilidades y el funcionamiento de mercados (altamente imperfectos), y que el Estado desempeña un papel muy decisivo en el mantenimiento y expansión del sistema capitalista, como asimismo, para asegurar la integridad del estado-nación.

La expresión global apunta a dos hechos. Tiene el significado más obvio y convencional del alcance mundial en un sentido "horizontal" o geográfico del sistema; lo que podría llamarse el "ensanchamiento" del capitalismo. Sin embargo, también hay una dimensión vertical, la "profundización" del capitalismo. El capitalismo transnacional no es sólo una manera especial de organizar el capital y la mano de obra en la producción y de apropiarse y utilizar el excedente. Además es un conjunto de ideas y creencias acerca de la sociedad (Sauvant, 1976a); un conjunto de organizaciones e instituciones que lo generan, desarrollan e imponen; y una comunidad global de hombres y mujeres que las adoptan; en sintesis, es también un sistema sociocultural". El "ensanchamiento" del

\footnotetext{
${ }^{5}$ La expresión es utilizada por Sorokin (1947 y 1962) como parte de su marco de referencia, pero el concepto está implícito en Polanyi (1944). Véase O. Sunkel y E. Fuenzalida (1974).
} 
capitalismo no impide que en muchos países subsistan en mayor o menor medida situaciones precapitalistas y semicapitalistas, pero siempre bajo el predominio de relaciones capitalistas de producción. Asimismo, la "profundización" del capitalismo no significa que haya penetrado en todos los aspectos de la vida social, en todas partes.

Por último, la frase en su nueva etapa de organización transnacional procura dar a entender que en la actualidad la principal característica del capitalismo oligopolico tecnoindustrial consiste en que la producción se organiza en un plano global más que nacional o local. Asi, las empresas transnacionales, que constituyen una elevada y creciente proporción del núcleo oligopólico de las economías capitalistas contemporáneas han repartido su estructura productiva a través del mundo, estableciendo filiales en numerosos paises (Magdoff, 1966; Levitt, 1970; Sunkel, 1970; Barnet y Muller 1974; Naciones Unidas 1973, 1974). En la actualidad, sus preferencias de localización se resuelven sobre la base del mercado mundial, cotejando, entre otros factores, los costos de la mano de obra, los subsidios e impuestos de los gobiernos, los tipos de cambio y la situación política de todos los países. La maximización de las utilidades también se logra en el plano global y respecto de la empresa en su conjunto. Esto se ha hecho posible porque las empresas transnacionales transfieren bienes intermedios y de capital, recursos financieros, tecnología, personal e información a través de las fronteras nacionales -en un plano transnacional-pero dentro de los límites de su organización. Con ello reemplazan en gran medida el mercado y por lo tanto disfrutan de relativa libertad para fijar precios, costos, honorarios y tasas de interés contables en sus transacciones con cada filial, de manera de maximizar las utilidades netas de la empresa mundial en su conjunto. Esta es la práctica que se conoce como fijación de precios de transferencia (Ellis y Joekes, 1977). Los precios, que se suponían variables independientes determinadas por las fuerzas del mercado, se han convertido para las empresas transnacionales en variables dependientes en un modelo contable de maximización global de las utilidades.

La tendencia de estas empresas es a independizarse del funcionamiento de los mercados como mecanismo relacionador de unidades de producción y consumo independientes, y reemplazarlos mediante la integración de estas distintas unidades dentro de una sola organización y bajo el mando de una sola estructura de decisiones, de tal modo que la planificación para toda la organización sustituya los mercados. Los productores y consumidores locales de las distintas regiones de un país acostumbraban relacionarse entre sí a través de los mercados, hasta que las empresas nacionales ocuparon el lugar de las empresas locales y crearon una red de filiales locales de la empresa nacional. Las empresas nacionales de un país acostumbraban vincularse con las empresas nacionales de otros países a través del mercado internacional, hasta que las empresas transnacionales de un país crearon filiales en otros o se apoderaron de las empresas nacionales de esos otros países y las convirtieron 
en filiales. Mientras que en la etapa internacional del capitalismo las economías nacionales interactuaban básicamente a través de los mercados (salvo el caso de algunos monopolios internacionales dedicados a la extracción y elaboración de productos primarios), en la etapa transnacional hay un creciente reemplazo de los mercados internacionales por transacciones dentro de la empresa transnacional.

Esta misma tendencia a avanzar de una organización descentralizada hacia otra altamente centralizada se está produciendo en otros planos de las relaciones internacionales: la generalización de las estructuras institucionales transnacionales formales e informales en los ámbitos de la política, la defensa y la seguridad interna, las finanzas, la cultura, las profesiones, los medios de comunicación, la información, la educación, la ciencia y la tecnología (Cruise O'Brien, 1979); (Fuenzalida, 1979); (Godfrey, 1979; Luckham, 1979).

\section{LOS PRINCIPALES COMPONENTES DEL CAPITALISMO TRANSNACIONAL}

\section{a) Las instituciones transnacionales.}

La institución dominante del sistema global es la empresa transnacional.

Es dominante porque centraliza las decisiones claves acerca de qué, cómo, cuánto,para quién y dónde producir; y dónde quiere decir ahora, en qué rincón del mundo. También es dominante porque, tanto individualmente como en su conjunto estas instituciones ejercen una poderosa influencia social, politica y cultural tanto nacional como global.

No obstante, las empresas transnacionales distan mucho de ser las únicas instituciones importantes del sistema global. Posiblemente son las más notorias debido a su enorme poder e influencia económica y política, los conflictos y confrontaciones envueitas con los Estados en que se han visto (Vernon, 1971; Naciones Unidas, 1973 y 1974;. Gilpin, 1976; Blair, 1977), y la enorme cantidad de recursos que consagran a promover su propia imagen (Sauvant, 1976a, 1976b). Sin embargo, hay toda una red de otras instituciones que contribuyen a facilitar las actividades de las empresas transnacionales. Algunas de ellas son instituciones intergubernamentales, como el Fondo Monetario Internacional (Wachtel, 1977), el Banco Mundial o la OCDE, en el campo económico, o la OTAN y el Tratado Interamericano de Asistencia Recíproca, en el militar, instituciones que son tan conocidas como las propias empresas transnacionales. Otras instituciones públicas y privadas, operan en los planos nacional o subnacional y tienen vínculos menos manifiestos en el sistema global, pero de hecho funcionan como parte de él. Pertenecen a esta categoría muchas de las que se dedican a la ayuda externa y a la promoción de las exportaciones, tanto civil como militar, y las actividades financieras.

Hay que atribuir especial importancia a las instituciones educacionales que capacitan al personal de las instituciones transnacionales. Se trata por lo general de destacadas universidades e institutos politécnicos 
de los países industriales y sus "filiales" académicas en el tercer mundo (Fuenzalida, 1971a)b), c); Vasconi y Reca, 1971, Leite Lopez,1972; Fagen, 1973; Alemann, 1974; Carnoy, 1974, Mazrui, 1975). Estas instituciones comparten con las revistas periódicas de circulación mundial, agencias noticiosas, empresas transnacionales de publicidad y redes de televisión, la labor de elaborar y difundir una concepción del mundo que implica la promoción de los intereses de las empresas transnacionales (Cruise O'Brien, 1974, Sauvant, 1976, Schiller, 1976; Smith, 1976; Somavia, 1976; Varis, 1976).

Por último cabe mencionar las revistas transnacionales académicas $y$ las redes informales de científicos y profesionales, cuyas reuniones $y$ conferencias periódicas son objeto de amplia publicidad y que no sólo ofrecen información básica, sino también el inventario de recursos humanos a partir del cual se contrata el personal especializado (Ben-David, 1968; Crane, 1972; Alger y Lyons, 1974).

\section{b) La comunidad transnacional.}

El sistema global es manejado por un estrato de la sociedad que se apropia de la mayor parte del excedente que produce. Su posición dentro de la estructura productiva abarca desde los dueños de los medios de producción y las personas que desempeñan los principales cargos administrativos y financieros, hasta los profesionales, técnicos y funcionarios de mayor jerarquía, tanto en las instituciones privadas como públicas.

La base de su hegemonía son sus conocimientos especializados y el hecho de que son indispensables para el proceso de crear y aplicar esos conocimientos a la producción de una sucesión de nuevos bienes y servicios, así como para el proceso de innovación tecnológica y de diferenciación de productos que es la principal razón de la superioridad de las empresas transnacionales.

Como se dijo, las actividades de las empresas transnacionales cuentan con el apoyo del progreso científico y tecnológico generado por las instituciones de enseñanza superior, y con el que le presta el personal altamente especializado al que éstas capacitan. Por lo tanto, la comunidad transnacional no se basa tan sólo en las empresas transnacionales, y en todos los sectores económicos en que éstas operan (industria, agricultura, mineria, transporte, construcción. comercialización, información, medios de comunicación, banca y finanzas, turismo, esparcimiento, etc.) sino también en las demás instituciones transnacionales (Field, 1971). Apoyándose en sus conocimientos especializados, su organización profesional y su prestigio social, estas elites ejercen cierto control sobre estas instituciones y tienen el poder de captar parte del excedente económico, lo que les permite mantener niveles de vida relativamente altos.

\section{c) La cultura transnacional}

El estrato de la sociedad que hemos llamado comunidad transnacional está formado por personas que pertenecen a distintos países, pero que 
tienen análogos valores, convicciones, ideas y una lingua franca, el inglés. También comparten modalidades de conducta notablemente similares en lo que toca a patrones profesionales, estructuras familiares, vivienda, modo de vestir, modalidades de consumo y orientación cultural en general (Sunkel y Fuenzalida, 1976). Así, pues, la comunidad transnacional comparte lo que podría considerarse una cultura transnacional. Como cualquier otra cultura, esta cultura transnacional tiene dos componentes principales: la cultura especializada y la común.

La primera surge de las actividades científico-tecnológicas especializadas que llevan a cabo los miembros de la comunidad transnacional, las que constituyen un elemento esencial para la expansión del capitalismo tecnoindustrial.

Este segmento de la cultura está impregnado de los conocimientos especializados que poseen sus portadores, conocimientos que son lo que les han permitido convertirse en miembros de la comunidad transnacional. Como estos conocimientos se basan en el progreso tecnológico alcanzado a través de la aplicación sistemática del llamado "método científico" a todos los aspectos de la realidad, su visión del mundo y de sí mismos se ve fuertemente influida por la convicción tecnocrática de que todos los problemas sociales son solucionables por medio del análisis racional y la aplicación del "método científico" (Gehlen, 1963; Schelsky, 1965; Ellul, 1967; Galbraith, 1967; Habermas, 1971; Berger, Berger y Keller, 1973; Steger, 1977).

El segundo componente de la cultura transnacional es la cultura común. El capitalismo transnacional ha influido en las costumbres, ideas, creencias, valores y comportamiento en materias tales como la vida familiar, la vivienda, las modalidades de consumo y otros aspectos de la vida cotidiana. Quizá uno de los efectos más importantes es el que produce en las modalidades de consumo, ya que la característica cultural principal de la dinámica del capitalismo oligopólico ha sido la creación de un mercado homogéneo de bienes y servicios de consumo a escala mundial. La comunidad transnacional es la expresión más completa y coherente de estas nuevas modalidades de consumo, pero ellas se difunden a través de sectores más amplios de la población como consecuencia del efecto demostración y de la utilización activa y amplia de los medios de comunicación (Villamil 1979 b). La consecuencia que ello tiene en las clases de menores ingreso es la adopción parcial de estas nuevas modalidades de consumo y la deformación de las existentes. Esto se refiere no tan sólo a los casos más conocidos relacionados con los bienes de consumo duraderos sino también a los alimentos básicos, por ejemplo cuando el pan sustituye al maíz o la mandioca (Barner y Müller, 1974; Brown, 1974; Sauvant, 1976, a, b).

\section{d) Organización espacial del capitalismo transnacional}

Las recientes revoluciones tecnológicas que han afectado el transporte y las comunicaciones han aumentado enormemente su velocidad y reduci- 
do drásticamente el costo de la distancia y del tiempo. La aplicación de innovaciones electrónicas a los procesos de producción ha permitido subdividir aún más el trabajo humano en tareas sencillas y claramente delimitadas. Por lo tanto, los procesos de producción han podido separarse crecientemente en etapas discretas y éstas pueden distribuirse a través de todo el mundo sobre la base de ventajas de localización tales como mano de obra barata y disciplinada, acceso a los mercados, subsidios gubernamentales, disponibilidad de recursos o insumos estratégicos y ausencia de reglamentación ambiental. En otros términos, la descentralización geográfica de la producción dentro y entre países puede acentuarse mucho más que antes para aprovechar al máximo las ventajas de ubicación, al mismo tiempo que se mantiene el control centralizado de la planificación, las finanzas, los recursos humanos, la comercialización, la producción y la innovación (Fröbel et al. 1977).

Como consecuencia de lo anterior tienden a producirse distintos tipos de configuraciones espaciales: por una parte, una separación funcional de las actividades administrativas, científico-tecnológicas y de producción de la empresa, en distritos urbanos financiero-administrativos, áreas de concentración de los establecimientos académicos y zonas industriales periféricas, respectivamente. Por la otra una descentralización jerárquica entre las sedes mundiales de las empresas ubicadas en ciudades tales como Nueva York, Londres, París, Frankfurt y Tokio; sedes regionales ubicadas en las ciudades de los países más transnacionalizados de las diversas regiones del Tercer Mundo, tales como Ciudad de México, Sao Paulo, Nairobi y Singapur; y sedes nacionales, usualmente en las capitales de países menos importantes o estratégicos (Hymer, 1971). A medida que se desciende del plano mundial a los planos local o nacional disminuye la posibilidad de formular políticas, planificar y tomar decisiones en forma independiente, y algunas funciones desaparecen por completo. Por ejemplo, las actividades científicas y tecnológicas innovadoras tendrán lugar casi íntegramente en el plano mundial, y lo mismo sucederá con la planificación estratégica global y la administración financiera y de los recursos humanos. Como es natural, esto entraña una creciente rigidez y una autonomía cada vez menor a medida que se avanza hacia la unidad local de producción, lo que es fuente de fricción y conflicto con el medio nacional o local.

En todos estos planos las instituciones transnacionales tienen que establecer contactos y relaciones con los gobiernos nacional o local, o ambos, con las empresas y con el mercado laboral tanto de profesionales, técricos y empleados administrativos como de mano de obra especializada. Con el tiempo, este proceso da lugar al desarrollo de instituciones y comunidades nacionales y locales de contrapartida, que tienden a integrarse cada vez más a las instituciones y comunidades transnacionales. Estas comunidades tenderán a concentrarse en zonas residenciales suburbanas y a reproducir la estructura urbana, las viviendas y los estilos de vida de las comunidades transnacionales de los países centrales. Los sectores de ingtesos 
medianos y altos de la población tratarán de imitar estos estilos de vida por medio de la adopción de las modalidades de consumo del sector transnacional.

Si bien los miembros de la comunidad transnacional que viven en los países centrales tienen mucho en común con aquellos que viven en los paises periféricos, hay una división del trabajo e importantes diferencias entre ellos. En lo que toca al primer aspecto, las personas que desempeñan los cargos de mayor jerarquía de las empresas transnacionales permanecerán en los países centrales puesto que conservan las funciones de concebir y de planificar y controlar su ejecución. Igual cosa sucede con la estrategia global y con la mayoría de los funcionarios que se ocupan de la innovación, tanto en el campo de la ciencia y la tecnología, como en los de la producción y la comercialización. En el plano local, las funciones de mayor jerarquía se limitarán a la administración y ejecución de las estrategias globales.

También surgen diferencias porque las élites transnacionales de los países en desarrolio se encuentran en una situación mucho más expuesta y segregada con relación al resto de la sociedad a que pertenecía y porque deben llevar a cabo las funciones de articular su país con el sistema global, a la par que conservar la integridad de su Estado-nación. Todo ello los lleva a vincularse más estrechamente con el Estado y a hacerse más expresamente políticas.

Como consecuencia de estas estructuras espaciales, los escasos recursos nacionales y locales se asignan de preferencia a ampliar los sectores "modernos" de pueblos y ciudades, lo que agrava al deterioro de los barrios más antiguos y la proliferación de los sectores marginales contribuyendo con renovada fuerza a la heterogeneidad caracteristica de las ciudades contemporáneas: modernas zonas residenciales, comerciales, gubernamentales, industriales e incluso de nuevas poblaciones obreras, entremezcladas con barrios residenciales, comerciales, de oficinas públicas, operarias e industriales más antiguos y en decadencia, todo ello rodeado de amplias barriadas marginales cada vez más extensas.

En sintesis, las instituciones y comunidades transnacionales se encuentran diseminadas en el espacio a través de todo el mundo en un archipiélago de núcleos de "modernidad", que se vinculan entre sí a través de una serie de estructuras jerárquicas centralizadas que definen núcleos dominantes y dependientes. Estos núcleos interactúan con las sociedades nacionales y locales, partes de las cuales se han integrado en mayor o menor medida con los núcleos transnacionales, mientras que el resto se ve afectado indirectamente a través del mercado de trabajo, el intercambio de bienes y servicios, los agentes socializadores constituidos por la educación y los medios de comunicación así como por la reasignación de los recursos a que dan lugar las influencias transnacionales.

Como el tamaño e importancia y tipo de núcleos transnacionales varía de país en pais, tal como sucede con las características. sociocultu- 
rales y políticas de las sociedades nacionales y locales, cada país presentará la misma semejanza genérica de la interacción entre un núcleo transnacional nuevo y en expansión y una sociedad preexistente, pero también acusará profundas diferencias según la naturaleza de la sociedad en que tiene lugar esta interacción. Por lo tanto, para comprender mejor el proceso de transnacionalización bajo distintos conjuntos de características nacionales, parece fundamental realizar estudios comparados de estos distintos tipos de situaciones. (Villamil 1979, b; Godfrey Langdon, 1979).

\section{e) El sistema transnacional y el estado nación}

El sistema transnacional ha desarrollado una infraestructura económica de empresas transnacionales $\mathrm{e}$ instituciones conexas, cuenta con una población formada por las comunidades transnacionales, estas comunidades comparten una cultura común y todos estos elementos se encuentran establecidos en un determinado territorio. Sin embargo, si bien estos elementos son parte integrante del sistema transnacional, también se encuentran bajo la jurisdicción de los estados nacionales. En otras palabras, el sistema transnacional se traslapa con un sistema de estados naciones. Como consecuencia de ello, los objetivos y procedimientos de ambos coincidirán en ciertos casos, pero en otros, cuando los objetivos y procedimientos de cada sistema sean diferentes, se producirán situaciones conflictivas (Naciones Unidas, 1973 y 1974; Senado de los Estados Unidos, 1977; Fortín, 1979; Langdon, 1979).

Desde el punto de vista de su racionalidad global, es probable que una empresa transnacional procure que la filial que posee en un país determinado produzca cierto artículo para el mercado local, empleando su propia tecnología, insumos y bienes de capital importados, comprando o desplazando a los competidores locales, manteniendo pleno control de su capital, administración y método de organización, utilizando sus propias prácticas de relaciones públicas y laborales y remitiendo el máximo de utilidades a la casa matriz, minimizando el pago de impuestos. Es posible, por otra parte, que ciertos gobiernos deseen fortalecer a los capitalistas nacionales, que la filial produzca para la exportación, que los insumos se adquieran en el pais, que sus nacionales participen en la propiedad y en la administración y que compartan los conocimientos técnicos, que la filial se adapte a las prácticas locales en materia de organización, contabilidad, y relaciones laborales, que reduzca al minimo sus remesas al extranjero, maximice la reinversión en el plano local y pague un impuesto razonable sobre las utilidades. Como es bien sabido, este es el conflicto que se produce tradicionalmente respecto de los requisitos que se exigen a la inversión privada extranjera. La cuestión en juego se refiere más que nada a la distribución del ingreso adicional generado por las filiales.

Un problema muchísimo más fundamental es el que se relaciona con la propia estrategia de desarrollo en su conjunto, la que está sujeta a una marcada influencia del núcleo transnacional local cuyo objetivo es repro- 
ducir localmente las condiciones de vida, modalidades de consumo y cultura que caracterizan a los demás núcleos transnacionales del sistema global. El principal instrumento a través del cual puede lograrse este objetivo es el Estado, de tal modo que el control, la influencia y el acceso al aparato estatal son cruciales. En los últimos decenios ello se logró como consecuencia de la expansión y transformación de las funciones del Estado a que dieron lugar las presiones internas y externas por alcanzar el desarrollo económico y social. Las nuevas actividades del Estado fueron marcadamente estimuladas por la asistencia técnica internacional que introdujo nuevos criterios y métodos en la administración pública y en la planificación. Surgió una nueva y más amplia tecno-burocracia gubernamental, tanto civil como militar, cuya función consistía en modernizar y racionalizar el Estado en su promoción del deșarrollo económico y social, como asimismo en su capacidad de habérselas con los conflictos internos y la subversión. (Luckham, 1979). Estos sectores sociales nuevos comparten en gran medida los valores, principios y métodos de la comunidad transnacional y tienen un interés directo en el proceso de transnacionalización. A través de su influencia global y local, y de su estratégica presencia interna dentro del propio Estado, la comunidad transnacional adquirió una significativa influencia sobre el proceso de asignación de recursos y formulación de políticas, bastante fuera de proporción en relación con su poder económico o político real. En su intento de reproducir en el plano local los métodos y estilos de vida de los paises desarrollados, han contribuido de manera importante a que se asignen recursos en gran escala a la satisfacción de estas "necesidades", lo que tiene efectos extremadamente positivos sobre el crecimiento económico y las condiciones de vida de una minoría de la población, incluidos ellos mismos, y consecuencias bastante menos favorables para la mayoría de la población.

La teoría tradicional sobre el desarrollo ha sostenido que no existe, tal conflicto, al menos a largo plazo, debido a que la expansión del núcleo "moderno" (transnacional) aumentará el producto total y, a su vez, esto producirá 'un "derrame" que, a su debido tiempo, mejorará la situación de la máyor parte de la población, como sucedió en el pasado en los paises actualmente desarrollados. Nuestro argumento, en cambio, es que el crecimiento capitalista transnacional contemporáneo ha estado dando lugar a una polarización intra e internacional cada vez mayor; lo que, a su vez, ha venido afectando las relaciones entre el estado nación y el capitalismo transnacional.

Los conflictos entre los estados y el capitalismo transnacional se mantuvieron en un segundo plano por la formidable expansión de este último hasta comienzos de los años setenta. Esta expansión dio la impresión de que existía una coincidencia de intereses entre ambos. Sin embargo, la crisis y la contracción económica de los últimos años ha hecho aflorar los conflictos latentes.

Entre los problemas de creciente gravedad que han debido confrontar los países desde 1.973 se cuentan la declinación de las tasas de crecimiento económico, la tendencia a un desequilibrio externo y fiscal cada vez mayor, 
el consiguiente incremento del endeudamiento y de las presiones inflacionarias, el aumento sostenido del desempleo, del subempleo y de la pobreza (Adelman y Morris, 1973) y el hecho de que cada vez hay menos margen de maniobra para las políticas redistributivas (Chenery et. al., 1974). Ello es particularmente grave en el caso de los países subdesarrollados pero también comienza a causar preocupación en los países desarrollados a medida que las empresas transnacionales efectúan cada vez mayores inversiones en el extranjero, estableciendo filiales manufactureras para atender a los mercados locales y para rexportar a los propios paises desarrollados. Como consecuencia de ello, las posibilidades de empleo se ven doblemente amenazadas y aumenta la presión política para que se adopten políticas proteccionistas. (Senado de los Estados Unidos, 1977).

Lo anterior ilustra las contradicciones entre la expansión del sistema transnacional y su necesidad de estabilidad política, tanto en los países desarrollados como en los subdesarrollados. En los primeros, donde el nivel promedio de vida es muy alto y la desigualdad menos marcada, las políticas de estabilización y de "ingresos" (incomes policies) han podido hasta ahora habérselas con los desequilibrios económicos de corto plazo. Sin embargo, a largo plazo existen problemas estructurales muy graves en relación al creciente desempleo estructural, al desequilibrio regional, al acceso a los recursos naturales, al medio ambiente y otros, que no dejan de estar vinculados con la expansión y características del capitalismo transnacional (Cole, 1977).

En los paises subdesarrollados, donde el nivel promedio de ingreso es bajo, la desigualdad es muy marcada y creciente y la pobreza ampliamente difundida, los profundos desequilibrios económicos y las agudas tensiones politicas sólo pueden refrenarse por medio de la fuerza. No puede caber mucha duda que la difusión de los regimenes autoritarios y militares que ha tenido lugar en los últimos años en los paises del Tercer Mundo se relaciona directamente con estas tendencias (Nun, 1969; Sunkel, 1972; O'Donnell, 1972; Touraine, 1976).

Las presiones objetivas ejercidas en los paises desarrollados y en los subdesarrollados porque se adopten políticas proteccionistas y nacionalistas han ido en aumento, pero al mismo tiempo se han limitado muchísimo más las posibilidades reales de lograr una mayor separación entre los procesos internos y los fenómenos internacionales. El sistema transnacional no sólo ha establecido fuertes vínculos estructurales de índole económica, social, cultural y politica a través de las fronteras nacionales, sino que ha creado un conjunto de organizaciones e instituciones internacionales tales como la OCDE, el Fondo Monetario Internacional, el Banco Mundial y la Comisión Trilateral, entre otros, que en conjunto constituyen algunos elementos de un estado global cuyo objetivo es mantener el sistema transnacional y hacer frente a cualquier amenaza a su funcionamiento. Por lo tanto, los "ajustes" sociopolíticos y económicos tienden a producirse en el plano interno más que en el internacional. 
El capitalismo tecnoindustrial, en su forma contemporánea de sistema transnacional global, es producto de un prolongado proceso histórico. Cada uno de sus componentes y sus interrelaciones son transformaciones de otros previos y coexisten y se interrelacionan con elementos que corresponden a etapas anteriores del desarrollo capitalista o de sistemas precapitalistas, o de ambos. Es conveniente reseñar brevemente dichas etapas para ayudar a comprender la fase porque atraviesa actualmente el desarrollo capitalista. Estos párrafos son mero esbozo de los principales períodos del desarrollo capitalista; existe al respecto una amplia bibliografia: además de los autores clásicos, últimamente han surgido otros que han analizado en forma más detallada la evolución del capitalismo como un sistema global (Polanyi, 1944; Ashworth, 1962; Hobsbawn, 1965, 1968, 1975; Davis, 1973, Braudel, 1974; Wallerstein, 1974 a,), asi como algunos autores que han relacionado en especial el desarrollo latinoamericano con la evolución del capitalismo global (Furtado, 1970; Stein y Stcin, 1970; Sunkel y Paz, 1970; Cortés Conde, 1973).

En relación con el pasado más distante, conviene recordar las siguientes etapas históricas principales: un periodo de formación del capitalismo como sistema comercial mundial, que abarca desde el último tercio del siglo XV al último tercio del siglo xvm (Trevor-Roper, 1959; Hobsbawn, 1965; Braudel, 1966; Davis 1973; Wallerstein, 1974a); la aparición.de los primeros casos históricos de capitalismo industrial y de naciones estados modernos en el último tercio del siglo Xvin y primer tercio del siglo xIx (Dobb, 1946; Hobsbawn, 1968; Landes, 1969, Mathias, 1969); la consolidación interna y la expansión imperial externa de los primeros estados capitalistas industriales, desde aproximadamente 1825 hasta el decenio de 1870 (Church, 1975; Hobsbawn, 1975), el capitalismo industrial como sistema global de comercio, finanzas e inversiones, en su etapa de organización internacional-imperial, entre 1870 y 1941 (Ashworth, 1962; Davies, 1973); la crisis del capitalismo internacional-imperial, el surgimiento de una alternativa socialista y los intentos de reorganizar el capitalismo industrial como reacción a èl de 1914 a 1945 (Polanyi, 1944); el capitalismo tecno-industrial global en su etapa de organización transnacional como reacción al periodo de crisis previo, y la consolidación interna y expansión externa de la alternativa socialista, desde aproximadamente 1945 a nuestros días.

El proceso de reorganización del sistema capitalista después del largo periodo de crisis comprendido entre 1914 y 1945 surgió de distintas situaciones nacionales. Los paises que habían sido liberados de la ocupación alemana por la Unión Soviética o que habian experimentado una revolución socialista, constituyeron el bloque socialista bajo el liderazgo de la Unión Soviética. Los países industrializados de Europa Occidental, sea vencidos (Alemania, Italia) o vencedores (Francia, el Reino Unido, Países Bajos, Bélgica) se vieron seriamente quebrantados por el esfuerzo bélico y tuvieron que hacer frente a problemas políticos internos muy 
graves ya que en ellos surgieron poderosos partidos de izquierda de índole reformista o revolucionaria. La situación del Japón en el Asia cra similar en algunos aspectos. En las colonias de los países industrializados de.Europa Occidental y Japón, en Africa, Asia y América Latina se agudizó la pugna nacionalista por alcanzar la independencia aprovechando la crisis de las potencias metropolitanas y la presencia de los países socialistas. Los Estados independientes no industrializados de esos continentes, en su mayor parte los de América Latina, habían adoptado políticas proteccionistas de industrialización basada en la sustitución de las importaciones en los años de crisis y de guerra y cjercían presión para que sus políticas se aceptasen y apoyasen en el plano internacional. Los Estados Unidos emergieron del periodo de crisis y guerra como la potencia económica, política y militar más poderosa del mundo y como el nuevo centro del sistema capitalista. En 1948 inició una ofensiva económica, diplomática, militar e ideológica en gran escala, destinada a detener el avance del bloque socialista y la amenaza de los partidos de izquierda en muchos paises capitalistas, tanto del centro como de la periferia. Se creó una red global de tratados internacionales de defensa reciproca entre los Estados Unidos $y$ los países situados en la frontera del mundo socialista y se mantuvieron o crearon bases militares estadounidenses en sus territorios.

Se estimuló la reconstrucción de las economias de los paises industrializados de Europa Occidental y de Japón a través de la transferencia masiva de recursos financieros y tecnología (Plan Marshall) y de la creación de la OCDE y de la Comunidad Económica Europea. En una ctapa posterior también se apoyó la industrialización y modernización de los países subdesarroliados especialmente de América Latina a través de la asistencia técnica, de la cooperación financiera y del fomento de la inversión privada extranjera. Por último se creó una red de organizaciones económicas, financieras $y$ de asistencia técnica internacionales destinadas a reconstruir el sistema capitalista internacional que se había desarticulado seriamente durante el periodo de crisis; desmantelar las estructuras $y$ políticas proteccionistas $y$ nacionalistas que habian adoptado todos los países en ese período; y promover la reintegración de los países subdesarrollados a ese sistema, una vez que se encontrase en marcha la reconstrucción y el resurgimiento del capitalismo curopeo.

Estos procesos, y algunas características fundamentales de la economia y de la sociedad estadounidense se cuentan entre las principales causas de la aparición del sistema transnacional.

La expansión territorial hacia el oeste de la economia y sociedad norteamericanas en el siglo XIX tuvo lugar en zonas escasamente pobladas a expensas de sociedades autóctonas relativamente primitivas y más que nada a través del subcontinente norteamericano, vasto territorio contiguo provisto de abundantes recursos naturales. La dimensión continental del país y su amplio mercado interno, asi como la escasez relativa de mano de obra, favorecieron el desarrollo de tecnologías de una elevada densidad de capital, la producción en gran escala y la gran empresa de am- 
plio alcance geográfico (Chandler, 1961). La ciencia, la tecnología y la actividad económica se habían vinculado estrechamente para contribuir al desarrollo de la producción en gran escala. Además, como consecuencia de la Gran Crisis, de la Segunda Guerra Mundial y de la Guerra Fría, que le siguió, que dieron lugar a una fucrte intervención del gobierno en la economía y a un gran esfuerzo bélico, se produjo una verdadera simbiosis entre el Estado, la gran empresa y la ciencia y la tecnología.

Este núcleo dinámico de la economía y sociedad estadounidenses, que hasta la Segunda Guerra Mundial había mantenido una posición relativamente "aislacionista" - salvo respecto del Caribe y Centroamérica- se extendió a través del mundo durante la guerra, y posteriormente, en el periodo de la Guerra Fría de los años cincuenta y sesenta, se mantuvo y amplió aún más. Esta fue la base de la expansión mundial del capitalismo tecnoindustrial estadounidense. Asimismo, fue fundamental para la reconstrucción y desarrollo de un "estilo" análogo de desarrollo capitalista en los antiguos centros capitalistas europeos y japonés, donde se formaron o reconstruyeron núcleos oligopólicos dinámicos similares, así como en los países en desarrollo, donde surgieron sectores "modernos" en expansión.

Pese a que el origen más inmediato del capitalismo transnacional son el sector oligopólico de la economía estadounidense, el establecimiento tecno-científico de la sociedad norteamericana, así como partes de su aparato estatal, su naturaleza "nacional" norteamericana ha ido mermando gradualmente a medida que han ido surgiendo centros dinámicos análogos de producción, ciencia y tecnología y Estado en los revitalizados centros industriales de Europa y el Japón, y a medida que sus filiales se amplían y penetran en los países en desarrollo e incluso en menor pero creciente grado, en los paises socialistas. Así pues, el propósito que tuvieron originalmente los Estados Unidos, de lograr la reorganización de un capitalismo en crisis bajo la hegemonía norteamericana, se ha ido transformando con el tiempo, convirtiéndose en una dinámica transnacional que a su vez está penetrando y afectando a la propia sociedad estadounidense, como también a todas las demás (Barnet y Müller, 1974).

\section{MECANISMOS DE INCORPORACION DE LOS PAISES SUBDESARROLLADOS AL SISTEMA GLOBAL}

Como el presente trabajo se ocupa principalmente del proceso de desarrollo capitalista en los países del Tercer Mundo el análisis se limitará a los efectos de la transnacionalización en estos países. Debido a que estos efectos dependen mucho de la forma en que se manifiesta el proceso de transnacionalización en cada caso, se examinarán ante todo los diversos medios a través de los cuales las sociedades nacionales interactúan con el sistema global: a) la inversión extranjera en los sectores de exportación de productos básicos, en el turismo o en el sector manufacturero, o en ambos, usualmente a través de la creación de filiales de las empresas transnacionales; con dos principales variantes en el caso del sector manufac- 
turero: producción para el mercado interno o para la exportación, en general a las sociedades industriales más avanzadas; b) la utilización sistemática de los medios de comunicación masivos (diarios, radio, televisión, cine), para crear demanda de nuevos bienes y servicios de consumo, à través del empleo de técnicas de publicidad avanzadas; en la mayoria de los casos esto entraña crear o ampliar y modernizar un sistema nacional de comunicaciones; c) el otorgamiento de préstamos y asistencia técnica internacional pública para racionalizar, modernizar y ampliar el aparato estatal, haciendo especial hincapié en la infraestructura económica y social $y$ en las instituciones militares; en los años más recientes ha existido además creciente acceso a un mercado financiero internacional privado en expansión acelerada; d) lo programas de becas, tanto civiles como militares, para capacitar funcionarios locales en las instituciones educacionales de los países centrales en las diversas ramas de la ciencia y de la ingeniería $y$ en las nucvas disciplinas de administración, planificación, cuentas nacionales, procesamiento de la información, medios de comunicación, comercialización, y "seguridad nacional" (Carnoy, 1974, Luckham, 1979); e) la reforma de las instituciones educacionales de tal modo de formar en el plano loca! recursos humanos capaces de reemplazar a los técnicos extranjeros (Fucnzalida, 197 Ic; Vasconi y Reca, 1971; Carnoy, 1974; Mazrui, 1975); f) la generalización de los critcrios, prioridades $y$ métodos del estilo transnacional a todos los aspectos de la vida social, desde las actividades económicas a la salud, la educación y la vivienda.

Estos diversos mecanismos han quedado de manifiesto en los distintos países en desarrollo desde comienzos de los años cincuenta. No comenzaron a operar en forma simultánea y fueron promovidos por distintos grupos sociales y profesionales, intereses economicos y dependencias gubernamentales, tanto de los países subdesarrollados y de los países industrializados. Sin embargo, aunque no fueron concebidos inicialmente para funcionar en forma simultánea y coherente, pronto quedó dc manifiesto que constituían una nueva actividad, un amplio proceso de innovaciones en su sentido más amplio: todo un conjunto de innovaciones que se apoyaban reciprocamente, en los campos de la producción, el consumo, la organización, el comportamiento, los valores, tanto del sector privado como del sector público; en sintesis, una nueva cultura. Durante la etapa internacional del capitalismo industrial el mecanismo de incorporación en el sistema global eran las exportaciones de productos básicos y la principal innovación tecnológica, el ferrocarril, además los grupos sociales afectados eran muy limitados. Como consecuencia de ello, la "modernización" era un fenómeno de "enclave". La modernización transnacional, con su énfasis en la creación de un mercado de consumo en gran escala, su dependencia de los medios de comunicación de masas, de las unidades locales de producción manufacturera de elevado coeficiente de capital y perfección tecnológica, de la acción gubernamental internacional planificada y de la reproducción en el plano local del "modelo" ofrecido por los países industriales, ha tenido efectos muchísimos más vastos, tanto deliberados como involuntarios. 
En general, los efectos son tres. El primero es la aparición de un núcleo transnacional dependiente en la sociedad subdesarrollada, encarnado en una comunidad local, con sus instituciones y cultura propias que se distingue marcadamente del resto de la sociedad y que controla en gran medida los mecanismos de un Estado cada vez más represivo.

Las instituciones que se integran a este núcleo incluyen las filiales de las empresas transnacionales, situadas en los distintos sectores de la economía; las empresas locales más grandes que utilizan tecnología avanzada, tanto privadas como públicas; los organismos especializados del gobierno que se ocupan de la planificación y ejecución de ambiciosos proyectos de desarrollo y de la planificación de areas tales como la salud, la educación, las ciudades, el transporte y las comunicaciones; las instituciones de educación superior, en especial las que se ocupan de la investigación cientifica; las organizaciones internacionales; las fuerzas armadas.

El conjunto de estas instituciones proporciona empleos, ingresos $y$ bienes y servicios a una parte de la población local que, por lo tanto, puedc compartir patrones de comportamiento análogos a los de los habitantes del núcleo dominante del sistema transnacional situado en los países desarrollados. Entre los patrones de comportamiento que llaman la atención de manera especial se cuentan los barrios residenciales de las capitales de los paises subdesarrollados que copian el diseño y los estilos arquitectónicos de zonas residenciales similares del núcleo dominante, y que tienden a estar fisicamente separadas del resto de la ciudad.

El segundo efecto es la creación de una masa creciente de personas desempleadas o subempleadas que, por tener fuentes de ingreso muy precarias e inestables, se ven obligadas a sobrevivir en condiciones de extrema pobreza, mientras que al mismo tiempo se las estimula insistentemente a aspirar a los niveles de vida de que disfrutan las personas del núcleo transnacional dependiente del sistema global. Esta situación es consecuencia directa de: a) la destrucción, desplazamiento o estancamiento de las instituciones socioeconómicas tradicionales que les ofrecían empleos, ingresos y bienes y servicios - por pobres y primitivos que fuesen-y su reemplazo por las instituciones más eficientes que trajo consigo el sistema global; b) la incapacidad de las nuevas actividades de alto coeficiente de capital de absorber el crecimiento demogránico de la fuerza laboral y el desempleo y el subempleo generados por la transnacionalización; c) el intenso esfiuerzo de los medios de comunicación por estimular el reemplazo de los bienes y servicios tradicionales por los productos de las nuevas actividades económicas, que se traduce en una modificación de la modalidad de consumo de la sociedad; $y$ d) la aceleración de crecimiento demográfico del sector más pobre de la población y de la fuerza laboral, como consecuencia de las innovaciones, modernización y ampliación de los servicios 
de salud, que han reducido en mayor proporción las tasas de mortalidad de los pobres que las de natalidad.

Como el proceso de transnacionalización es básicamente urbano, esta masa de personas marginadas se concentra en zonas situadas en torno a las grandes ciudades, $y$ principalmente en la capital, donde se exhiben profusamente los bienes y servicios a que se aspira, en dramático contraste con las escasas posibilidades de empleo. Allí se encuentran fisicamente segregadas del resto de su propia sociedad. El violento contraste entre lo que les depara su destino de marginales y el de los que han encontrado un lugar en el nuevo sistema, y la escasez objetiva de formas "legítimas" de obtener los bienes y servicios deseados, los lleva a reaccionar de diversas maneras, en contra de esta situación, tanto individual como colectivamente. A su vez, las acciones de los desposeídos hacen reaccionar a los privilegiados, que recurren al Estado para asegurar su protección. Como estos sectores controlan en buena medida al Estado, la fuerza de éste se utiliza contra las masas empobrecidas, contribuyendo a su naturaleza autoritaria. Como se indicó, los efectos de transnacionalización y polarización antes descritos tienen una tercera consecuencia: que es la acentuación de la naturaleza autoritaria y represiva del Estado a medida que aumenta el peligro que se cierne sobre los grupos sociales hegemónicos. Sin embargo, también hay otra clase de reacciones que procuran hacer frente a las fucrzas causales y no sólo a sus consecuencias. Estas reacciones son los intentos de cortar los vínculos de la sociedad dependiente con el sistema global y reorganizarla de manera menos polarizada, tanto en lo que toca a la acción como al pensamiento sociales. Estos intentos son un efecto secundario de los dos antes mencionados y van desde el redescubrimiento y la reformulación de los valores, de los símbolos, del arte y en general de la cultura nacional hasta la creación de movimientos políticos cuyo principal objetivo es lograr una mayor autonomía de la sociedad, o de partes de ella, del sistema global (Oteíza y Sercovic, 1979; Villamil, 1979c).

Así pues, el proceso de transnacionalización tendría tres dimensiones: la integración transnacional, la desintegración nacional y los intentos de reintegración.

\section{OBSERVAGIONES FINALES}

Las observaciones relativas a las consecuencias de la incorporación al capitalismo mundial afectan a todos los países, pero el grado de permeabilidad y la intensidad con que se sienten estas consecuencias varían de país a país. La evolución histórica de cada uno, su forma de incorporación al sistema global tanto ahora como en las etapas anteriores del desarro-llo capitalista (incluída su experiencia colonial) influirán en el grado e intensidad de estas consecuencias. Además pueden señalarse una serie de otras características de la mayor importancia: tamaño, ubicación y dotación de recursos naturales, alcance del proceso de industrialización, naturaleza y organización de la fuerza laboral, importancia del Estado y 
evolución y fuerza de una tradición cultural y de instituciones "no occidentales".

Cada país combina estas variables de diferente manera pero, en general, los paises podrian clasificarse al respecto en algunos tipos principales:

a) Países relativamente grandes, en que capitales extranjeros y nacionales explotan sus recursos naturales para la exportación, que están situados a "prudente" distancia del poder hegemónico, que se caracterizan por un proceso avanzado de industrialización, con una fuerza laboral urbana relativamente organizada, con un Estado intervencionista y un grado avanzado de formación de una cultura nacional basada en instituciones culturales propias. (Por ejemplo, algunos países sudamericanos tales como Argentina, Brasil, Chile, Colombia, Venezuela).

b) Países pequeños, con escasa dotàción de recursos naturales, situados cerca de la potencia hegemónica, con un débil proceso de industrialización, escaso grado de organización de la fuerza laboral, cierto grado de urbanización, un Estado débil y una cultura nacional muy disminuida (Puerto Rico y la mayor parte del Caribe).

c) Países medianos, bien provistos de recursos naturales, que aún no se han explotado plenamente, con un proceso de industrialización en ciernes, bajo nivel de urbanización, experiencia colonial e independencia recientes, Estados y cultura nacional débiles, pero fuertes culturas tribales (por ejemplo Kenia).

d) Países grandes, con escasos recursos naturales, situados a gran distancia de la potencia hegemónica, con cierto grado de industrialización. Ciudades muy populosas pero básicamente rurales, estado y cultura nacional débiles, pero con una cultura preindustrial fuerte $\mathrm{y} \cdot \mathrm{rica}$, con sus propias instituciones (por ejemplo, la India).

Como es natural, estas categorías son muy generales y amplias y su enumeración tiene propósitos meramente ilustrativos. Lo que hay que destacar es la necesidad de estudiar más a fondo las experiencias de los distintos países, pues sólo a base del conocimiento de casos específicos es posible iniciar la formulación de estrategias y políticas de desarrollo destinadas a superar la integración transnacional y la desintegración nacional. 
Auelman, I. $y$ Talft Morris, C., 1973, Economic Growth and Social Equity in Developing Countries, Stanford University Press, Stanford, California.

AlemanN, H, von, 1974, "International Contacts of University Staff Members; Some Problems in the Internationality of Science" International Social Science Journal, Vol 26. $\mathrm{N}^{\circ} 3$, p. 445-457.

Al.Ger, C.F. y Lyons, G.M., 1974, "Social Science as a 'Transnational System", en International Social Science Journal, Vol. xxvi, No 1.

AMIN, S., 1972, Accumulation on a World Scale, Monthly Review Press, New York.

1973, Le Développement Inégal. Essai sur les Formations Sociales dit Capitalisme Péripherique, Les Editions de Minuit, Paris.

AsHWORth, W., 1962, A Shart History of the Initernational Economy 1850-1950. Longmans, London.

Aston, T. (ed.), 1965, Crisis in Europe 1562-1660, Routledge and Kegan Paul.

BLaIR, J.M., 1976. The Control of Oil, Macmillan, London.

BARNet, R.J., y Müller, R.E., 1974, Global Reach: The Power of the Multinational Corporations, Simon and Schuster, New York.

BEN-DAvID, J., 1968, "Nationa! and Internationa! Scientific Communities". Manuscrito no publicado, Hebrew University, Jerusalem, Isracl.

Berger, P.; Berger, B. Y Kellner, H., 1973, The Homeless Mind, New York, Vintage Books.

BERNSTEIN, H. (cd.), 1973, Underdevelopment and Development, Hammordsworth, Penguin Books Lid.

Braudel, F., 1966, La Mediterranée et le Monde Méditerranéen á l'Epoque de
Philippe n, 2e. ćd., 2 vol. Lib. Armand Colin, Paris.

1974, Capilalism and Material Life, 1406-7800, Fontana Collins, Great Britain.

Bkows, L., 1974, By Bread Alone, Praeger, New York.

Cardoso, F.H. and Faletto, E., 1971, Dependencia y Desarrollo en América Latina, Siglo Xxı1, México.

Cakposo, F.H. 1974, "Notas sobre el Estado Actual de los Estudios sobre la Dependencia" en J. Serra (ed.) Desarrollo Latinoamericano. Ensayos Críticos. Fondo de Cultura Económica, México.

CArnoy, M., 1974, Education as Cultural Imperialism, New York, David McKay.

Cepal, 1951, Economic Survey of Latin America, 19.49 United Nations, New York.

Chandoler, A.D., 1961, Strategy' and Structure, Chapters in the History of the Industrial Enterprise, Doubleday and Co., New York.

Chenery, H., et al., 1974, Redistribution with Grouth, Oxford University Press.

Снurch, R., 1975, The Great Victorian Boom, 1850-1873, Macmillan, London.

Cule, S., 1977, Global Models and the Inter. notional Economic Order, Pergamon Press, Oxiord.

Cortes Conde, R., 1973, The First Stages of Modernization in Spanish America, New York, Harper and Row.

Crune, D., 1972, Invisible Colleges, The University of Chicago Press, Chicago.

Cruise O'Brien, R., 1974, "Dominance and Dependence in Mass Communications: Implications for the Use of Broadcasting in Developing Countries", IDS Discussion Paper, $\mathrm{N}^{\circ} 64$. 
CRUISE O'Bries, R., "Mass Communication: Social Mechanisms of incorporation and Dependence", en Villami] (ed.) $1979 \mathrm{a}$.

Cuev^, A., 1976, "A Summary of Problems and Perspectives of Dependency Therory", Latin American Perspectives Issue 11, Fall, vol. III, $\mathrm{N}^{\circ} 4$.

Davis, R., 1973, The Rise of the Atlantic Economies, Weidenfeld, London.

Dовв, M., 1946, Studies in the Development of Capitalism, Routledge and Kegan Paul, London.

Dos Sartos, 'T., 1968, El Nuevo Carácter de la Dependencia, Cuadernos del Centro de Estudios Socio-Económicos de la Universidad de Chilc, Santiago.

1969, "The Crisis of Development Theory and the Problems of Dependence in Latin America", en Bernstein, H., 1973 .

Ellis, F. Y Joekes, S., 1977, A Report of the Procedings of the IDS-UnCTAD Conference: Intra-Firm Transactions and their Impact on Trade and Development, iDs, 7 th-11 th November.

Eudu:, J., 1967, The Technological Sociely, New York.

Emamanuel, A., 1972, Unequal Exchange: A Study of the Imperialism of Trade, Monthly Review Press, New York.

Facen, P.W., 1973, Chilean Universities: Problems of Autonomy and Dependence, Sage Comparative Politics Series, Bcverly Hill, California, Sage Publications.

FIEL,D, J.A. Jr., 1971, "Transnationalism and the Ncw "Tribe" en Keohane, R.O. y Nyc, J.S. (cd.), 1971.

Fortis, C., 1979, "The State, Multinationa! Corporation and Natural Resources in Latin America", en Villamil (cd.), 1979a.

FoXLEY, A., (ed.), 1974, Distribución del Ingreso, Fondo de Cultura Económica, México.
Frank, A.G., 1967, Capilalism and Underdevelopment in Latin America, Monthly Review Press, New York.

Fronel., F., Heinrichs, H. y Ḱreye, O., 1977, Die newe internationale Arbeitsteilung, Rowohlt, Taschenbuch Verlag Gmbh, Reinbek bei Hamburg, Germany.

Fuenzadida, E., 197 Ia, "Problemas de Ciencia y Tecnologia en el Paso al Desarrollo", Mensaje, xx, Septiembre Octubre, pp. 444-451.

1971b, Investigación Científica y Estratificación Internacional, Santiago, Andrés Bcllo.

1971c, "La Dependencia en el Ambito del Saber Superior y la Transferencia de Modelos Universitarios Extranjeros en América Latina", en Modemización y Democratización en la Universidad Latinoamericana, Santiago, Corporación de Promoción Universitaria, p. 3-27.

Fuenzalida, E., "The Problem of Technological Innovation in Latin America", en Villamil (ed.) 1979a.

Furtado, C., 1970, Economic Development of Latin America; A Survey from Colonial Times to the Cuban Revolution, Cambridge University Press.

1973, "The Concept of Exiernal Dependence in the Study of Underdevelopment" in Ch. K. Wilber (cd.) The Political Economy of Development and Underdevelopment, Random Housc, New York.

Gacbrarth, J.K., 1967, The New Industrial State, Boston, Houghton Mirllin.

Gehlen, A., 1963, "Ueber Kulturelle Kristallisationen" in Studien zur Anthropologie und Soziologie, Berlin.

GilpIN, R., 1976, U.S. Power and the Multinational Corporation: The Political Economy of Foreign Direct Investment, Macmillan, London.

Girvan, N., 1973 "The Development of Dependency Economics in the Caribbean and Latin Amcrica: Review and Comparison" Social and Economic Studies, Vol. 22. $N^{\circ} 1$, March. (Special 
Issue on Dependence in the New World and the OId).

Godfrex, M., 1977, "Surplus Population and Under-Development: Reserve Army or Marginal Mass?", Manpower and Employment Research, Vol. 10. $\mathrm{N}^{\circ} 2$, April, McGill University, Montrea!.

GODFREY, M., 1979, "The International Market in Skills and the Transmission of Inequality" in Villamil (ed.) 1979a., y Langdon, S., "Partners in Underdevelopment? The Transnationatization Thesis in a Kenyan Context" en Villamil (ed.), 1979a.

Habermas, J., 1971, Toward a Rational Society, London, Heineman.

Hobsbawm, E.J., 1965, "The Crisis of the Seventeenth Century", in T. Aston (ed.), 1965.

1968, Industry and Empire. An Economic History of Britain Since 1750, Weidenfeld and Nicolson, London.

1975, The Age of Capital, 1848-1875, Weidenfeld and Nicolson, London.

Hymer, S., 1971, "The Multinational Corporation and the Law of Uneven Development", in J. Bhagwati (ed.), Economics and World order, Macmillan \& Co., New York.

INKeles, A., 1975;, "The Emerging Social Structure of the World", en World Politics, 27, July, pp. 467-495.

Keohane, R.O., y Nye, J.S. (eds.), 1971, Transnational Relations and World Politics, Harvard University Press, Cambridge, Mass.

1977, Power and Interdependence, Little, Brown \& Co., Boston and Toronto.

Landes, D., 1969, The Unbound Prometheus. Technological Change and Industrial Development in Western Europe from 1750 to the Present, Cambridge University Press.

LANGdon, S., "Multinational Corporations and The State in Africa" en Villamil (cd.), 1979a.
Leite Lopes, J., 1972, La Ciencia y el Dilema de América Latina: Dependencia o $\mathrm{Li}$ beración, Bucnos Aires, Siglo XXI.

LEvITT, K., 1970, Silent Surrender, The Multinational Corporation in Canada, Macmillan of Canada, Toronto.

Lickнам, R., "Mititarism and International Dependence: A Framework for Analysis", en Villamil (ed) 1979a.

Magdoff, H., 1966 The Age of Imperialism, Month Review Press, New York and London.

Mazru1, A., 1975, "The African University as a Multi-National Corporation: Problems of Penetration and Dependency", Harvard Educational Review, Vol. 45, $N^{\circ}$ 25, May', pp. 191-210.

Meister, A., 1975, "Le Système Transnational", Civilisations, Vol. $\mathrm{xxv}, \mathrm{N}^{\circ} 1 / 2$, pp. 33-51.

Mennis, B., y Sauvant, K.P., 1976, Emerging Forms of Transnational Community, D.C. Heath and Company, Lexington, Mass.

Mrehalet, C.A., 1976, Le Capitalisme Mondial, Paris, Presses Universitaires de France.

Morse, E.L., 1976, Modernization and the Transformation of International Relations, The Free Press, New York.

MURray, R., 1971, "The Internationalization of Capital and the Nation State", New Left Review, N 67, May-June.

Nun, J., 1969, "Superpoblación Relativa, Ejercito Industria! de Reserva y Masa Marginal", Revista Latinoamericana de Sociología, Vol. $5, N^{\circ} 2$.

O'Brien, P., 1975, "A Critique of Latin American Theories of Dependency", en I. Oxaal, T. Barnet y D. Booth (eds.), Beyond the Sociology of Development, Routicdge and Kegan Paul Ltd.

O'Donnell, G.A., 1972, Modemización y' Autoritarismo, Editoria! Paidos, Buenos Aires. 
Otelza, E., y Sercovic, F. "Collective Self -reliance: Some OId and New Issues", en Villamil (ed.) 1979a.

Pailoix, C., 1973, Les Firmes Multinationalés et le Proces d'Internationalisation, Maspero, Paris.

PinTo, A., 1976, "Styles of Development in Latin America", en CEPAL, Review, First Semester.

and Kr̃akal, J., 1973, "The Center-Periphery System 20 Years Later", Social and Economic Studies, March.

Polanyt, K., 1944, The Great Transformation, Farrar, New York.

Prebisch, R., 1949, "The Economic Development of Latin America and its Principa! Problems", Economic Bulletin for Latin America, Vol. vin, N 1 , February 1962 (publicado originalmente en 1949). 1976, "A Critique of Peripheral Capitalism", en CEPAL Reviez, First Semester.

Quijano, A., 1974, "The Marginal Pole of the Economy and the Marginalised Labour Force", Economy and Society, Vol. $3, N^{\text {ro }} 4$, November.

Sauvant, K., 1976a, The Potencial of Multinational Enterprises as Vehicles for the Transmission of Bussiness Culture" in Controlling Multinational Enterprises: Problems, Strategies, Counterstrategies, K. Sauvant y F. Lavipour (eds.), Boulder, Colorado, Westview Press, 1976.

1976b, "His Master's' Voice", CERES, Vol. 9, $N^{\circ}$ 5, September-October, 1976, pp. 27-32.

$y$ Mennis, B., 1977, "Puzzling Over the Immaculare Conception of Indifference Curves: The Transnational Transfer and Creation of Socio-Political and Economic Preferences". Paper presented to the Second German Studies Conference, Iridiana University, Bloomington, April.

Schelsky, H., 1965, "Der Mensch in der wissenschaftlichen Zivilisation", en - Auf der Suche nach Wirklichkeit, Duss-Koln, Diederichs, 1965.
Scherer, F.M., 1971, Industrial Market Struclure and Economic Performance, Rand McNally, Chicago.

SChiller, H., 1976, Communication and Cultural Domination, New York, International Arts and Sciences Press, 1976.

Smatr, K', 1976, "The Impact of Transnational Book Publishing on Intellectual Knowledge in Less Developed Countries". Presentado a una reunión de Expertos, Unesco, Paris, ist-5th June, 1976. SHc-76/Conf. 635/10.

SOMAviA, J., 1976, "The Transnational Power Structure and International Information" en Development Dialogue, $N^{\circ} 2$, pp. 15-28.

Sorokis, P., 1962, Social and Cultural Dynamics, New York, The Bedminster Press. 1947, Sociely, Culture and Personality, New York, Harper and Brothers.

STEGer, H.A., 1977, "Dominación Transnacional y De-cualificación Regional del Intelecto: La Vinculación entre los Regimenes "Consulares" y la Reforma Universitaria en América Latina", Papers, N 7, Barcelona.

Stein, S. y Stein, B., 1970, The Colonial Herilage of Latin America, New York, Oxford University Press.

SUNkEl, O., 1967, "Dependencia Externa y Política Nacional de Desarrollo", Revista de Estudios Internacionales, Vol. 1, $\mathrm{N}^{\circ}$ 1, Santiago-Chile.

1971, "Capitalismo Transnacional y Desintegración Nacional en América Latina" en Estudios Internacionales, $\mathrm{N}^{\circ} 4$, enero-marzo.

1972, "Big Business and Dependence" en Foreign Affairs, April.

1974, "External Economic Relationships and the Process of Development: Suggestions for an Alternative Framework of analysis", en Williamson, Glade and Schmitt (cds.), Latin AmericanU.S. Economic Interactions, American Enterprise Institute for Public Policy Research, Washington.

1977, "El desarrollo de la teoría del de- 
sarrollo", Estudios, Intemacionales, Año X, $N^{\circ} 40$ (octubre-diciembre).

y Fuenzalida, E., 1974, Transnationalization, National. Disintegration bs Reintegration in Contemporaty Capitalism, ins lnternal Working Paper $\mathrm{N}^{\circ} 18$. y Fuenzalida, E., 1976, "The Effects of Transnational, Corporations on Culture". Trabajo presentado a una Reunión de Expertos, UNEsco, Paris, June, SHC-76-Conf. 635/6.

Y. Paz, 1970, El Subdesarrollo Latinaamericano y la Teoría del Desarrollo, Siglo xxi, Editores, México.

Tayares, M.C., y Serra, J., 1971, "Más Allá del Estancamiento", Revista Latinoamericana de Ciencias Sociales, junio-diciembre.

Touralke, A., 1976, Les Societés Dependantes, Essais sur l'Amérique Latine, Sociologie Nouvelle, Paris.

Thevor-Roper, H.R., 1965, "The General Crisis of the Seventeenth Century", en T. Aston, 1965.

U.N. DEPT, OF ECONOMIC ASD SOCIAL. Afrairs, 1973, Multinational Corporations in World Development, (ST/ECA) 190 and Con. 1), New York.

1974; The Impact of Multinational Corporations on Development and on International Relations (E/5500/Rev. 1, St/ ESA/6), New York.

United States Senate, Committee on FoREIGN RELATIONS, 1977, International Debt, the banks, and U.S. Foretign Policy, A Staff Report, foreword by Senator Frank Church, Us, Government, Printing Office, Washington.

United States Senate, 88th Congress, 2nd Session, Pursuant to Resolution 262, 1964, Economic Concentration: Hearings Before the Subcommittee on Antiirust and Monopoly of the Committee on the Judiciary, in particular statements by Mueller, W., Blair, J. and Adelman, M.A.
Varsos, C.V., 1974, Intercountry Income Distribution and Transnational Enterprises, Clarendon Press, Oxford.

VAris, T., 1976, "The Impact of Transnational Corporations on Communication". Trabajo presentado a Reunión de Expertos, unesco, Paris, June. SHC/conf. $635 / 7$.

VAscon, T.A., y Reca, I., 1971 , Modernización y Crisis en la Universidad Latinoameticana, Santiago, Cuadernos del Centro de Estudios Socio-Económicos de la Universidad de Chile.

VAlenzUEla, J.S.; Y ValenzUela, A., "Modernization and Dependence: Competing Paradigms in the Study of Latin American Development" en Villamil (ed.), 1979 a.

Vernon, R., 1971, Sovereignty at Bay: The Multinational Spread of U.S. Enterprises, Basic Books, Inc., New York.

VillakiL, J.J., (ed.) 1979a, Transnational Capitalism and National Development, Harvester Press, Hassocks, U.K. 1979. 1979b, "Puerto Rico 1948-1976: The Limits of Dependent Growth" en Villa* mil (ed,) 1979a.

1979c, "Planning for Self, Reliant Growth" en Villamil (ed.) 1979a.

WaChtel, H.M., 1977, The New Gnomes: Multinational Banks in the Third World, Transnational Institute Pamphiet $\mathrm{N}^{\circ} 4$, Washington, Amsterdam.

Wallerstein, I., 1974a, The Modem World -System, Academic Press, New York, San Francisco, London.

$1974 \mathrm{~b}$, "The Rise and Future Demise of the World Capitalist. System: Concepts for Comparative Analysis", Comparative Sludies in Society and History, xvi, $N^{\circ} 4$, pp. 381-415.

1976, "From Feudalism to Capitalism: Transition or Transitions? Social Forces, $\mathrm{L}, \mathrm{V}, \mathrm{N}, 2$. 